\title{
Belphégor
}

\section{Populaire, infiniment parlant (texte annoté par Lise Dumasy)}

\section{Charles Grivel and Lise Dumasy}

\section{(2) OpenEdition}

1 Journals

\section{Electronic version}

URL: https://journals.openedition.org/belphegor/1387

DOI: $10.4000 /$ belphegor.1387

ISSN: 1499-7185

\section{Publisher}

LPCM

\section{Electronic reference}

Charles Grivel and Lise Dumasy, "Populaire, infiniment parlant (texte annoté par Lise Dumasy) ", Belphégor [Online], 16-1 | 2018, Online since 21 August 2018, connection on 19 October 2021. URL: http://journals.openedition.org/belphegor/1387 ; DOI: https://doi.org/10.4000/belphegor.1387

This text was automatically generated on 19 October 2021

\section{(c) (i) (9)}

Belphégor est mis à disposition selon les termes de la Licence Creative Commons Attribution - Pas d'Utilisation Commerciale - Pas de Modification 4.0 International. 


\title{
Populaire, infiniment parlant (texte annoté par Lise Dumasy)
}

\author{
Charles Grivel and Lise Dumasy
}

\section{REFERENCES}

Paru originellement dans Jacques Migozzi (dir.), Le Roman populaire en question(s), Limoges, PULIM, « Littératures en marge », 1997. La version présente a été annotée par Lise Dumasy, Professeure à l'Université de Grenoble. Les notes de sa main sont précédées de l'indication $\mathrm{NdE}$ (note de l'éditeur).

$1 \quad 1^{1}$

2 "Populaire" fait plonger au plus profond des actions qui le caractérisent - dans l'infinité de ces actions, dans l'excès affolant et singulier qui de plus en plus les marque et nous les représente ${ }^{2}$.

3 "Populaire" : la matière est tragique, la matière est logique. Pour moi, le phénomène implique en effet une "masse" - le nombre des récits qui circulent et la compulsion incommensurable des données analogues à laquelle ils se livrent -, mais cette quantité - "industrielle" - n'entraîne pas par soi-même la perte d'aura - le déclin de quelque valeur que ce soit - et ne signifie nullement que la "littérature de consommation" s'épuise en la vaine répétition de ses données stéréotypes de base ${ }^{3}$ : certes, la répétition caractérise, par excellence, ce que nous appelons "populaire", mais cette répétition, à mon sens enfonce sous l'écorce le clou du délire (ou celui du désir ?)-un imaginaire devient mien, qui ne l'était pas, le voici greffé à même, au-dedans de mon esprit, comme le substitut de celui-ci : je suis mentalement un homme truqué. Dévalorisation, aliénation sont des termes impropres à signifier cela.

4 Le roman populaire, par quoi il faut entendre la forme tragique de la communication littéraire en régime industriel, par opposition à sa forme lyrique, que propose la chanson, - encore une fois, pour "dégradé" qu'il soit - fixe l'insu, arrête le regard sur ce qui échappe au regard, comme un art de l'inconscient qu'il est ${ }^{4}$. Un message est 
avidement proféré, mais retiré. Une émotion violemment ressentie, puis effacée. Un geste tenté, puis repris, puis retouché, comme un essai d'être agi par lui, y compris pourtant la gêne qu'il occasionne - le bonheur qu'il procure, avec son amour, le malheur qu'il engendre, avec son amour aussi. La donne est celle-ci: l'ignorance implique la connaissance, les cartes narratives professant l'une aussi bien que l'autre sur chacun de leurs deux côtés.

Le roman populaire propose un écran sur lequel on se fascine ${ }^{5}$ - la reconnaissance, de personne à personne - $\mathrm{y}$ est à la fois due et indue. Ces nœuds passionnels, ces intrigues emmêlées, ces enlèvements de femmes, ces séductions de mâles, ces tourments et ces soupirs, cette exaltation dans le mal, cette élévation dans les bons sentiments, cette dérogation perpétuelle, cette espérance qui ne parvient pas à se clore, ces irruptions et ces larmes, ce sang et ces membres qu'il faut aller jeter ou aller ramasser, cette mort enfin qui rôde par délégation d'un être malfaisant, son couteau à la main dans la nuit, et ainsi de suite au tréfonds de nos croyances, tout cela absorbe et tout cela répugne, figé en face au-dedans de la page, indu aussi par la vision de la lecture à l'esprit qui s'y porte, admiré et rejeté, connu et inconnu, congru et incongru, objet d'une enquête mais objet aussi d'une tentative de dissimulation, complète l'une aussi bien que l'autre. Car le lecteur est, comme chacun sait, à la fois le policier et le criminel et la page a deux versants, celui de la transparence et celui de l'insondable obscurité. Ce double volet de l'histoire étant précisément ce qui la rend intéressante. En un mot, ces histoires que dévide le roman populaire, on les sait et on ne les sait pas. Un suspens nous fait désirer arriver à leur fin - ah ! éteindre enfin le feu de l'histoire ! -, mais une insistance nous attache à elles et nous fait renvoyer à plus tard leur inéluctable clôture -ce savoir d'avant tout savoir enfin possédé dont elles montrent bien qu'il ne sera jamais ni confié, ni conquis. Prométhée est bien scellé à son rocher.

6 René Guise écrivait en $1986^{6}$ : "Le champ à explorer est immense et le domaine populaire encore fort mal balisé " : le constat est toujours pertinent, preuve en est, par exemple, le fait, ${ }^{7}$ tout ensemble caractéristique et affligeant, que les catégories, je dirais: usées, qui ont servi des générations durant à appréhender la littérature des élites - ou la littérature avec un grand L, comme on voudra -, continuent à remplir leur office dans ce cadre neuf. Or, on sait que l'histoire littéraire trouve son fondement dans une solide tradition et que celle-ci présuppose l'existence de normes constitutives des genres : un "roman" est à ses yeux moins un modèle de communication qu'un objet grammatical et rhétorique achevés; la valeur qu'on lui reconnaît dépendant directement de l'explication des règles qui sont censées être les siennes. A ce compte, la littérature populaire, plus souple et moins regardante, est toujours perdante. Par contre, ce qui lui confère son très remarquable dynamisme - son sans-gêne, son côté provocant, son humour à la fois fin et gras, sa coloration parodique mais aussi tragique, cette façon qu'elle a de jouer sur les deux tableaux, et beaucoup d'autres choses encore -, demeure pour une large part sous-exposé : la matérialité feuilletonesque de l'écriture, la sérialité et la prolixité, la visualité "in-texte" (mentalisée) et hors-texte (illustration), comme aussi le phénomène éditorial et tout ce qui touche à la professionnalisation de l'écrivain (le fait qu'il reçoive des honoraires et l'anonymat croissant que requiert la réglementation de son travail). Par une sorte de fatalité, l'étude populaire suit les mêmes et prévisibles voies que l'étude du littéraire - avec quelques tours de retard. Il faudrait donc redresser le cap - c'est à quoi je tâcherai de m'employer dans ces pages. 
7 Un autre point mérite encore l'attention. René Guise toujours, notre père à tous en archéologie populaire, notait, dans le même article, qu'un certain travail de dépoussiérage" en la matière devenait urgent ${ }^{9}$. Il avait en vue les idées préconçues et les a priori, légion dans ce domaine, qui encombrent la route. Mais la poussière est aussi d'une autre qualité : elle est celle qu'on trouve déposée dans les tombeaux encore intacts. Même si la littérature populaire (la culture de masse) ${ }^{10}$ n'est pas une autre grande Thèbes, elle est pourtant l'objet d'une certaine profanation. Nous, spécialistes, universitaires, amateurs, sommes des violeurs de tombes. Car si tout savoir suppose qu'on ait entrepris une archéologie de l'objet d'étude - qu'on l'ait décomposé strate par strate -, ce dernier pourtant fait résistance et tend à se dérober à la prise : les produits $\mathrm{du}$ langage ont tous leur secret - ou bien il était prévu, dans l'institution, que la recherche s'en mêle et dès lors ils ont pris leur précaution, ou bien ils étaient censés n'offrir la matière d'aucun savoir et dès lors ils donnent d'eux-mêmes accès, en toute candeur, à leurs méthodes et à leurs structures. Or, cette immédiateté s'est révélée très vite un leurre : un texte populaire n'est pas plus transparent que tel ouvrage disponible sur un autre circuit, ses facilités sont fallacieuses, ses intrigues à double fond, sa conclusion indécidable et sa fonction, si l'on y regarde bien, énigmatique. En tout état de cause, l'archéologue déblaie une tombe qui n'est pas profanée et il devra recourir à toute sa perspicacité s'il veut décrypter le chiffre enfoui de messages qui n'étaient pas faits, en principe, pour être compris par lui. Car le mystère d'un fait de langue tient aussi à celui que soi-même on emploie et l'obscurité d'un phénomène gît pour commencer dans l'œil qu'un spectateur, pour avisé qu'il soit, braque sur lui.

\section{2}

8 Je ressaisis quelques fils qui me paraissent tenir des Réflexions que je proposais à TapisFranc numéro 1, opuscule à peine paru de l'hiver 1988, avec quelques autres obligeamment prêtés malgré eux par les spécialistes. Cela formerait, en dix points, en matière populaire, mon bréviaire actuel ${ }^{11}$ :

9 1. La littérature populaire, singulièrement le roman, s'adresse à un appétit que ne calme aucunement «l'autre littérature ». Cette demande - ce feu ! - est excitatif; il me consume. Cette demande ne s'embarrasse d'aucun protocole, aucun protocole ne la brime: je lis ce que je veux lire - sans réticence ni scrupule. A même les figures quelquefois les plus involontaires. En toute inconnaissance de cause, droit devant, avec la volonté déterminée d'en finir. Je suis, ainsi, populairement fébrile.

10 2. Je constate un mouvement pendulaire : il n'y a pas, d'un côté, le populaire, et, de l'autre quelque chose qui ne le serait pas, le para - ou le péri - de ce qui serait, hors eux-mêmes, leur centre, le placé bas de ce qui serait inversement le haut, un impropre combiné à ce qui serait son mieux - et son gage -, mais seulement deux Etats de jouissance unique, tour à tour acquiesçante et refoulée. Qu'un objet "plein" de quelque nature qu'il soit, de langage par exemple, est impropre à contenter. "Populaire" signifie ainsi d'abord que la jouissance (de lire, qui est déclenchée dans lire) n'est ni une, ni unique - au contraire, ce que je lis la multiplie, et puis la dissémine.

11 3. La littérature populaire ne fait pas plus dans l'imaginaire que l'autre, que nous croyons plus maîtrisée. L'imaginaire n'est pas une autre case que la réflexion: je lis Dumas (le père) ou Jean Ray ou Leroux (qui sont mes frères) sans incohérence excessive. Je pense dès que je les lis. Je lis donc ie suis. 
12 4. La littérature populaire n'est pas plus orale que l'autre. Sauf à remonter à des temps qui ne connaissaient justement pas de littérature. Je m'inscris donc en faux, quoique amicalement, contre ce qu'affirme, lui aussi, Alain-Michel Boyer dans un article récent qui voudrait clarifier la question : "N'y a-t-il pas de nombreuses correspondances entre la littérature des peuples sans écriture et la paralittérature des sociétés de masse ?", "la paralittérature s'apparente aux contes et aux mythes de la tradition orale", "la paralittérature jouerait le rôle de la tradition orale dans les sociétés de masse", "la paralittérature s'est réappropriée certains mécanismes d'une rhétorique née justement dans le cadre d'une culture orale ${ }^{12}$. Mais on ne "cause" pas plus chez Dumas que chez Diderot ou Proust - bien qu'on en entretienne la fiction, et bien que le dialogue passe pour être le socle communicationnel du récit de l'auteur des Trois Mousquetaires. Il faut préciser encore que cette supposée oralité n'inscrit nullement l'écriture industrielle dont je parle dans la lignée du conte, et, pour ce qui concerne son analyse, sous le signe de Propp : les fonctions propiennes sont anéanties dans l'exposé narratif du feuilleton et de la série étant donné leur mode de parution et il faudrait être aveugle aux ruses agencées par la fiction pour reconnaître ici un merveilleux à l'œuvre. Quoiqu'il en soit, la littérature qui émerge au début du siècle passé est écrite et un texte la constitue : son "oralité" est donc jouée sur les pages, "parler" simule la vérité du récit - ce qui n'est pas l'un des moindres de ses attraits. Quand je parle, je m'adresse; quand j'écris, je travaille sur le délai de cette adresse ; mais quand je feins de parler mon récit, je fais l'économie mentale du support et me précipite - ou précipite mon lecteur - sur le terrain de l'immédiate té heureuse qui s'appelle le réel. Mais, bien sûr, la conviction qui m'anime en cela est un leurre.

13 5. La littérature populaire n'est pas plus - mais pas moins non plus ! - issue de la création collective que l'autre, il n'y a pas d'échappatoire à la collectivité en littérature - si tant est que celle-ci désire se faire lire. Certes, le travail du texte, la mise en œuvre, la gravure stylistique, en un mot: l'écriture caractérise le livre et détermine une part de son destin institutionnel. Mais il n'y a pas d'écriture détachée des croyances de la communauté - que ce soit celle que constituent entre eux les intellectuels ou, que ce soit celle des gens du peuple. J'ai montré dans un travail ancien déjà - Les Créances Duras. Compilation des universaux de texte ${ }^{13}$ - de quelle nature et de quelle remarquable prégnance était le savoir ordinaire transporté (ou impliqué) au niveau microscopique par une œuvre littéraire du réseau dit cultivé - Dix heures et demie du soir en été, de Marguerite Duras, un ouvrage qui à sa parution, en 1950, chez Gallimard, n'est certainement pas populaire justement. De même, il me paraît plus que risqué d'assigner à l'unique régime populaire la pratique de l'écriture d'atelier et celle du nègre. (Soit dit en passant, on regrettera qu'Alexandre Dumas fasse, et lui presque seul, les frais de la démonstration, dans la grande presse comme chez les spécialistes. ${ }^{14}$ ) Je ne crois pas que le texte populaire se fonde par principe dans l'indétermination générale. Je ne crois pas qu'il tende plus qu'un autre à l'anonymat - qu'on songe seulement à la longue série des auteurs ayant reçu le prix Goncourt : la très grande majorité sont retournés à l'oubli exactement comme il en va de la plupart de ceux qui écrivent pour les masses. Il ne convient donc pas de créditer la littérature en elle-même (il vaudrait mieux dire l'écriture) $)^{15}$ - populaire ou pas - des effets qu'engendre à son propos l'institution. L'anonymité du texte et la platitude du style existent en littérature populaire comme ailleurs : il y a un style Féval, un style Jean Ray, et - bien sûr ! - un style Dumas, inimitable et admirable. Par contre, ici comme là, Dupont reste Dupont. Je rappelle enfin, que de toute façon, l'auteur n'est jamais bien celui qu'on pense; homme de 
l'œuvre - ce pantin ou ce portrait-robot - est un produit de l'école ; qui écrit, comme disait Barthes ${ }^{16}$, est toujours déjà un peu mort. Invisible, inassimilable et mort.

6. Il faut poser en principe que les mécanismes inhérents à la textualité valent en littérature populaire comme ailleurs, et donc qu'écriture il y a, bonne ou pas. Je ne crois pas, pour emprunter encore une fois une expression à A.-M. Boyer, que la "paralittérature" puisse apparaître fondamentalement "comme une écriture de l'extériorité, niant même l'écriture comme expression individuelle", ni qu'elle soit foncièrement antithétique à la notion d'auteur - notion, comme on sait, parfaitement multiple - ou à celle d'œuvre - guère unitaire, elle non plus, si l'on considère l'Histoire littéraire ${ }^{17}$. Je ne partage donc pas le point de vue de Daniel Couégnas, selon lequel un ouvrage est paralittéraire de ce qu'il propose "un programme simple pour une lecture univoque" et qu'il n'obéit à aucune esthétique ${ }^{18}$. La substance populaire n'est ni simple, ni pauvre. Elle n'est pas pourvue d'un ego défaillant. Il n'est pas conseillé de chercher à la comprendre hormis son support : les mêmes histoires émergent de livre en livre, de bande en bande, à l'infini c'est certain, mais sans jamais signifier la même chose. Le populaire ne se définit pas plus et pas moins qu'autre chose par sa dimension "idéologique" (comprise ici avec une valeur négative comme ce qui ne relève pas de la sphère individuelle, voire s'y oppose) : c'est un vieux refrain que Sainte-Beuve déjà nous chantait. Mais c'est un refrain intéressé. On ne criera pas trop vite, toujours s'agissant de populaire, au "monosémique" - à l'"univocité - à la "fermeture" - à la "saturation" du programme narratif. ${ }^{19} \mathrm{Ce}$ qu'on s'efforce de caractériser sous ces termes est plutôt la médiocrité - ou la besogne ordinaire -, et cela à tous les niveaux de production des textes - il y a de mauvais ouvrages d'avant-garde tout aussi "clos" que de mauvais bouquins de kiosque de gare : simplement, ils le sont, mauvais, les uns et les autres, autrement.

Du reste, il ne s'agit pas que de cela. Le populaire - infiniment parlant - convient au brassage symbolique. Tout y est possible et dicible, tout y arrive, et pour de vrai - c'est là son charme. La littérature, la bonne, à tous les étages, comprend dans son principe la distance vis-à-vis de ce qu'elle énonce : elle est ironique et s'emploie à caricaturer aussi les vérités qu'elle applique.

Cela revient à dire qu'il n'existe pas d'en-soi de la littérature. On trouve des ouvrages rétrogrades et pires, c'est même l'évidente majorité, faisant feu de tout bois et tirant sans scrupules sur les ficelles les plus éprouvées - à tous les niveaux de consommation, cultivé aussi ${ }^{20}$. Cette manière de dire (et de voir) n'est pas l'apanage du circuit populaire. Une certaine culture bourgeoise et scolaire implique en soi le goût du rabâchage et magnifie volontiers la recette éculée: ne nous étonnons donc pas d'en avoir tous les jours, ici comme là, en haut comme en bas, sous les yeux l'affligeant spectacle.

Sur la distraction. Rien ne délasse vraiment: littérature populaire, littérature des intensités. Ce que je fais en entrant dans le registre du noir. (Le noir des uns est le rose des autres). Ce que j'éprouve quand je change de régime. Quand je change la gamme et passe au non penser : certainement pas je me délasse. Rien ne divertit vraiment de rien - la tête est trop prise. Quelqu'un court après les représentations qui lui sont nécessaires mais qu'il ne saurait avoir. Il croit, bien entendu, et ne croit pas, bien entendu, à ce qui lui est transmis; il s'identifie autant qu'il ne s'identifie pas. Il compense sans compensation. Il sublimise sans sublimation. Le commerce littéraire peut cela. Le populaire tombe à pic: il offre toutes les solutions possibles, à la fois et 
dans tous les sens - il les offre, mais il les retire. Opium? Oui, opium, mais dans un sens assez peu voisin de celui du vieux père Marx. Car, dans quel l'état est-on plutôt de veille ? ${ }^{21}$ Là où l'on se trouve réellement ou là où l'on ne se trouve décidément pas? Il n'existe pas de registre "fictionnel". La fiction agit premièrement de ce côté-là de nos vies.

8. Il n'y a pas de "tapis-franc" en littérature, qu'elle soit "populaire" ou quelle ne le soit pas : le "jeu" avec les mots de la langue et l'esprit qu'émettent ces mots n'est jamais pleinement licite ; une "franchise" en écriture contient nécessairement plusieurs états. Prenons pour exemple le très beau travail de traque proposé par Francis Lacassin à propos de la véritable identité de l'abbé Faria et sur l'origine réelle de l'histoire dont Dumas fait le récit dans le Comte de Monte Cristo ${ }^{22}$. La réalité, plus ou moins triturée, du fait historique n'est pas ce dont il s'agit 1) puisque nous lisons, le sachant, un roman, et 2) parce que cette supposée réalité historique, nous ne la connaissons pas quand nous lisons. La connaîtrions-nous, d'ailleurs, qu'elle ne nous émouvrait pas.

L'outrance, qui est volontiers populaire, est une catégorie positive de représentation. On ne semble guère regarder, populairement, aux moyens à employer pour parvenir à ses fins : mais il faut que ces moyens soient gros, spectaculaires, démonstratifs. S'ils sont cela, ils sont bons. Je note l'intempérance du récit, je note le goût pour les contrastes un peu voyants : la vraisemblance des actions ici est secondaire, pourvu qu'elles chargent bien. Populaire, c'est mélo, trappes et coïncidences, beaucoup d'argent, action grandiose et bel amour. Populaire, c'est à l'excès : un coup de couteau ne suffit pas si le meurtrier peut aussi en porter sept. Car sept tue bien plus certainement par l'impression dramatique qu'il laisse. Populaire, l'encre rage! Zombies, diaboliques, psychopathes héros hantent ces lieux, pas de place pour la ménagère - sinon dans les peaux successives qu'elle revêt. Car vie ordinaire et songe extravagant du livre se situent du même côté de l'incroyable barre que pose écrire.

On se rappelle le texte du sentencieux prospectus publié en 1853 par les éditeursimprimeurs de la Bibliothèque des poètes et romanciers chrétiens que reproduit Michel Nathan dans Splendeurs et Misères du roman populaire ${ }^{23}$ : "le génie du mal a recruté une armée de poètes et de romanciers dont le talent a contribué plus qu'on ne pense à l'explosion des révolutions qui sont venues périodiquement affliger la France, etc." La scène choisie pour écrire est en effet souvent, sinon toujours la pire ; on la préfère pour elle-même - dans tout ce qu'elle recèle d'impropre et de nécessaire et aussi pour le pouvoir déflagrateur qu'elle comporte. C'est que le pire, par excellence, est dramatique; son immoralité" (?) vient peut-être principalement des performances qu'il permet d'accomplir. C'est le spectaculaire qui est le déterminatif et non la "cause" que l'on défend ou le "contenu" qui préside à cette cause : qui lit entend principalement jouir jouir en premier lieu du spectacle présenté, puis jouir - en second lieu - de la conformité qu'il aperçoit entre ce qu'il voit et ce qu'il croit. Disons même que l'intensité des scènes "inconvenantes" ou violentes tend à décoller le sens de l'image perceptive - ainsi qu'un rouge intense au moment d'un incendie. On ne sait pas ce qu'il faut croire d'un incendie.

21 10. La répétition est belle. Je ne demande que ça : que ça continue et recommence. Ce qui frappe, dans le populaire, c'est la perfection à laquelle atteint le mécanisme de répétition. La variation existe - ce n'est jamais à cinq heures la même marquise, mais son air ne trompe pas. Lancinance, qui est belle, des représentations populaires. Un désir de pérennité trouve ici enfin à se satisfaire, un désir de redondance et de 
rengaine. La stéréotypie, loin d'être une marque de défectuosité, remplit son plein office. On ne dépasse pas - Lautréamont l'avait bien vu - la beauté du stéréotype. Y a-til quoi que ce soit à comprendre qu'un stéréotype ne formulerait pas?

Populaire : non pas seulement pour tous, de tous, allant à tous, mais au fond de tous ${ }^{24}$. Part recélée et part inquiète. Michel Nathan, dans son Lautréamont, notait la chose ainsi: "Le feuilleton touche à l'essentiel: l'amour, la mort, le désir, la violence, les tabous, la manière de les vivre et de les dire"25. Voici pensé "populairement" en moi ce que je ne peux penser, ce qui fait problème d'être pensé, ce que je m'astreins pourtant, contre moi-même, à penser en le lisant dans l'infini développement narratif des livres. Le refoulement invite au ressassement - et c'est tant mieux. La compulsion fait avaler des milliers de substances - et c'est tant mieux. L'une des bonnes vieilles règles conversationnelles de Grice ${ }^{26}$ prévoit qu'un énoncé n'est à prendre au sérieux, et donc à assimiler pour tel, qu'autant qu'on peut supposer qu'il s'efforce de dire la vérité qu'il vise. Mais un ouvrage de la sorte que nous disons ne se mesure pas à la vérité qu'il proclame, ni au but patent qu'il poursuit. Son sérieux - s'il existe - n'est pas la preuve de son sérieux. D'ailleurs, l'esprit du lecteur se délecte de toutes les invraisemblances qu'on lui donne en partage et symbolise sur ce terrain-là à outrance.

Populaire est la matière mère. Sa substance gît au fond de l'"autre" roman et réciproquement -, comme elle gît au cœur de tout sujet de lecture possible. Nous collons tous nos matériaux - dévalués, peu importe; nous faisons de la récupération mentale. J'en prendrai pour preuve, pour finir, les paragraphes 144 et 145 - "bifurcation A". "Le Monstre de Strasbourg" - de la sorte d'écrit autobiographique publié par Jacques Roubaud sous le titre de :La Boucle en 1993. ${ }^{27}$ L'être populaire est là, sous l'irrécusable nom de Rocambole: Rocambole - une identité qui se dérobe - une infinité de destins aléatoires - substitutifs du mien propre $^{28}$ ("lui" joue ma partie quand je lis) permettant de prendre la mesure de l'immesure, ici de l'amour - tout autant de l'échec (il va falloir recommencer l'histoire, la mener jusqu'à ce même terme, et plus loin encore) - tout autant de l'interdit (le message à moi adressé est à moi aussi refusé, le père et la mère s'étant donné le mot pour parvenir à cette fin-là) - tout autant de l'oubli (j'ai beaucoup lu, je crois l'avoir lu, je ne peux que l'avoir lu, que j'en aie abandonné ou pas la lecture, c'est une destinée). Qu'on aille y voir!

\section{NOTES}

1. $\mathrm{NdE}$ : Disposition en segments, (de 1 à 9) très fréquente chez Charles Grivel. On peut y voir le refus d'un dispositif plus académique - trop « conforme ", voire conformiste, trop ennuyeux, pas assez impliquant pour le scripteur comme pour le lecteur. Plaisir d'une conversation que l'article lui aussi doit aviver. Le lecteur est appelé à compléter le dispositif réflexif que le scripteur met en place. Assertion du singulier.

2. $\mathrm{NdE}$ : Dès le premier paragraphe, plusieurs identifiants du roman populaire pour Charles sont immédiatement présents : actions, infinité, excès, singulier.

3. $\mathrm{NdE}$ : Reprise de la question de la répétition et du stéréotype, mais sous un autre angle que celui sous lequel ils sont habituellement considérés : désir, délire, dont le lien à la répétition et au 
stéréotype est ici affirmé, sans être pour autant systématiquement exploré, ce qui est aussi une marque de fabrique de Charles : la perche au lecteur est tendue, à lui de la reprendre, s'il le veut. Art de l'effleurement, de la suggestion, qui rend le texte désirable justement parce qu'il ne dévoile pas complètement.

4. $\mathrm{NdE}$ : Belle définition, et peu fréquente, du roman populaire, qu'on aimerait voir plus développée, ces regrets font partie aussi de ce que les textes de Charles, souvent, suscitent. Ils appellent à des développements : à vos écritoires, Mesdames et Messieurs !

5. $\mathrm{NdE}$ : Le spectacle, l'image, l'écran - plutôt que la scène de théâtre, malgré la référence à la tragédie, sont des termes qui reviennent souvent sous la plume de Charles Grivel. L'écran est aussi bien celui du cinéma - que le roman populaire anticipe, attaché comme lui au fantôme, autre objet insistant de la théorie grivellienne qui se construit au fil des pages - que celui du fantasme, qui en lui se confond, comme le montre la suite de ce long et beau paragraphe.

6. NdE : Un hommage appuyé est rendu par Charles Grivel dans cet article à René Guise, trop tôt disparu (1994). Les deux hommes étaient très différents, tant dans la vie que dans leur approche du roman populaire. Mais pour moi qui les ai bien connus tous les deux, je peux dire qu'ils s'estimaient vraiment, et se reconnaissaient, malgré ces différences.

7. René Guise, Recherches en littérature populaire. Textes réunis par Christiane Guise. Dans : TapisFranc (Publication des Amis du Roman Populaire) nº 6 (1993-1994), p.ll.

8. $\mathrm{NdE}$ : Charles mettait - à juste titre à mon sens - en cause les définitions catégorielles des genres, toujours reconstruites a posteriori, et éminemment dépendantes de normes socialement et historiquement constituées. L'intéressait plus l'échange vivant dont le livre lu est le support. D'où son intérêt pour les théories de la communication, qui nourrissaient aussi sa réflexion, et dont on trouve de nombreuses traces dans les termes employés; d'où aussi une approche du roman populaire radicalement autre, adaptée au phénomène lui-même.

9. Op. cit. p. 13

10. $\mathrm{NdE}$ : Il y a beaucoup de Charles aussi dans cette désinvolture avec laquelle il dresse sans (paraître) y songer, en passant, une équivalence sur laquelle beaucoup s'interrogeront : peuple, masse : veut-on dire la même chose ? Renvoyant à l'indifférence cette distinction qui a pourtant fait couler beaucoup d'encre - Et il a raison de le faire. Car dans sa perspective, cette différence n'existe pas, ou n'est pas décisive. Elle est un fait de catégorisation externe, qui n'a pas d'impact sur le type d'analyse qu'il mène...

11. $\mathrm{NdE}$ : Les «dix points» du «bréviaire actuel » de Charles Grivel « en matière populaire ». Certains points déjà abordés ailleurs par Grivel y sont rappelés: l'appétence, la fébrilité de lecture du roman populaire: le cru de cet acte qu'est la lecture d'un roman populaire, la jouissance, débridée ou refoulée selon l'objet de lecture, mais la même, l'unicité du sujet qui s'y donne, s'adonne à l'imaginaire, mais aussi à la réflexion dans la lecture - la populaire comme l'autre, la constitution du sujet par et dans la lecture : «L'imaginaire n'est pas une autre case que la réflexion (...). Je lis donc je suis ».

En fait tous les points de ce bréviaire sont issus de la même conviction : il n'y a pas de différence essentielle entre littérature populaire et littérature du "réseau cultivé » : l'une " n'est pas plus orale que l'autre» (p. 527) «n'est pas plus - mais pas moins non plus ! - issue de la «création collective que l'autre » (p. 528). Bref, «il n'y a pas d'en-soi de la littérature » (p. 531), mais « des effets qu'engendre à son propos l'institution » (p. 529, et qu'il importe de ne pas confondre avec la réalité des pratiques de celle-ci).

Cette démonstration, qui démonte bien des stéréotypes souvent reçus sans examen, me paraît convaincante, pourvu qu'on y ajoute - ce qu'esquisse Charles - ce qui différencie fonctionnellement les textes: par exemple le fait qu'ils s'appuient sur les croyances d'une communauté plutôt composée d'intellectuels, ou plutôt composée de gens du peuple.

12. Alain-Michel Boyer, "Questions de paralittérature". Dans : Poétique $\mathrm{n}^{\circ} 98$ (avril 1994), p. 133, 135,142 et 143. 
13. Dans Ch. Grivel et J.A.G. Tans (ed.) Recherches sur le roman 1, Publications de l'Université de Groningue, CRIN 1-2 (1979), p. 73-138.

14. C'est aussi la lecture d'A.-M. Boyer, op. cit., p. 135-136. Même façon de voir tout récemment encore chez Umberto Eco et Pérez-Reverte. (J'ai essayé de montrer comment il fallait tenter de tordre le cou à la légende dans "Alexandre Dumas : mal écrire, bien écrire". (P. Bassan, Cl. Schopp (ed.), Les Trois Mousquetaires, Le Comte de Monte-Cristo: Cent cinquante ans après, Marly-le-Roi, Champflour, 1995, p. 188-199)).

15. NdE : « La littérature... (il vaudrait mieux dire l'écriture) (p. 529) « la textualité » (p. 530) : ces concepts barthésiens sont ceux que privilégie Charles Grivel en général, parce qu'ils permettent de considérer le texte dans son rapport individualisé à la réception, sans brouillages externes qui font de la littérature et de l'auteur sans qu'on s'en avise parfois des objets sociaux, historiques, éminemment idéologiques. En tant qu'objet textuel, en tant qu'écriture, la distinction entre «bon» ou "mauvais» ne passe pas entre littérature populaire et littérature quoi d'ailleurs? savante? cultivée? poétique ? bourgeoise ? ou, comme il est écrit un peu plus loin (p. 530) : « Il y a de mauvais ouvrages d'avant-garde tout aussi «clos» que de mauvais bouquins de kiosque de gare : simplement ils le sont, mauvais, les uns et les autres, autrement. » Les concepts d'écriture et de textualité sont utiles à Charles (et à nous...) en ce qu'ils permettent de ne pas mettre les questions là où elles ne sont pas : «Il faut poser en principe que les mécanismes inhérents à la textualité valent en littérature populaire comme ailleurs, et donc qu'écriture il y a, bonne ou pas » (infra).

16. ${ }^{6}$ La Mort de l'auteur". Dans : Manteia 5 (1968), p. 12-17.

17. Op. cit., p. 138-139.

18. Introduction à la paralittérature, Seuil, 1992, p. 154 et 85 ("L'esthétique paralittéraire n'existe pas").

19. Ibid., D.120 et 125 .

20. NdE : Rien de plus vrai que ce constat, pourtant rarement fait : la médiocrité et le rabâchage ne sont pas l'apanage de la littérature populaire à laquelle on les attribue en général - manière peut-être de fermer les yeux sur toutes les autres manifestations de la médiocrité, de cette façon nous mettant dans l'incapacité de véritablement analyser ce qui fait cette médiocrité, où qu'elle se produise, mais affectant le populaire automatiquement de l'indice "médiocrité ", ce qui est une opération très idéologiquement connotée. La crudité des attaques de la caste cultivée (et bourgeoise) contre le roman populaire au XIX ${ }^{\text {ème }}$ siècle ne laisse aucun doute là-dessus...

21. NdE : Souvent dans la pensée de Charles, un concept, mis en question - et ce sont souvent les plus employés qui sont mis en question parce que justement ils ne le sont pas ailleurs - convoque la mise en question du couple qui le définit dans l'opposition. Pour dire que la littérature populaire nous distrait, nous délasse, sait-on donc ce qu'est cet état de "veille " auquel le délassement s'oppose? Qu'est-ce qu'est réellement la veille? Mode d'interrogation proprement philosophique, parce qu'il ravive l'interrogation qui devrait porter sur le cœur même de la langue, pour libérer la réflexion de l'usure habituelle des concepts repris sans examen. Démarche créatrice aussi. Charles était convaincu qu'un travail critique était tout autant un travail d'écriture qu'un travail poétique, ou fictionnel, et que donc le travail du langage devait y répondre aux mêmes exigences de création. Cette conviction le rend parfois abscons, mais elle contribue le plus souvent à donner leur force de stimulation intellectuelle à ses textes.

22. "L’Abbé Faria ou Pèlerinage en Lourdes de la littérature populaire". Dans : Fr. L., Passagers clandestins, tome I, U.G.E. 10-18, 1979, p. 65-97.

23. ${ }^{11}$ Presses de l'Université de Lyon, 1990, p. 21-22.

24. $\mathrm{NdE}$ : Le mot est lâché : «tous » le «populaire » ne qualifie pas ce qui convient à, ou s'adresse à la masse, le peuple, etc... mais à cette indifférenciation du tous - tous sans exception, chacun de nous - sans exclusion, parce qu'il s'adresse à ce qui est au fond de tous : le désir, le refoulé, qui ressurgit dans le ressassé : « le refoulement invite au ressassement ». 
25. 1992, p. 10.

26. NdE: Dans la linguistique, c'est à la pragmatique que s'intéresse essentiellement Charles Grivel, parce que ce qui l'intéresse est ce qui se passe, se produit, dans le processus de lecture.

27. Pages 405 à 408 .

28. NdE : par le travail en amont qu'elle implique - de réflexion et d'écriture tout à la fois, la formule grivellienne percute, comme la flèche qui se fiche au milieu de la cible et c'est là peutêtre le plus précieux apport de Charles Grivel, un apport que l'on pourrait qualifier de maïeutique, en ce qu'il vise à solliciter, susciter, faire fructifier, accoucher, chez son interlocuteur - lecteur - une pensée. 